\title{
Comparative RNA-Seq analysis reveals a critical role for brassinosteroids in rose (Rosa hybrida) petal defense against Botrytis cinerea infection
}

\author{
Xintong Liu ${ }^{1+}$, Xiaoqian Cao ${ }^{1 \dagger}$, Shaochuan Shi ${ }^{1}$, Na Zhao ${ }^{1}$, Dandan $\mathrm{Li}^{1}$, Peihong Fang ${ }^{1}$, Xi Chen ${ }^{2}$, Weicong Qi ${ }^{3^{*}}$ \\ and Zhao Zhang ${ }^{1 *}$ (D)
}

\begin{abstract}
Background: One of the most popular ornamental plants worldwide, roses (Rosa sp.), are very susceptible to Botrytis gray mold disease. The necrotrophic infection of rose petals by B. cinerea causes the collapse and death of these tissues in both the growth and post-harvest stages, resulting in serious economic losses. To understand the molecular basis of rose resistance against $B$. cinerea, we profiled the petal transcriptome using RNA-Seq technology.

Results: We identified differentially transcribed genes (DTGs) in petals during B. cinerea infection at $30 \mathrm{~h}$ post inoculation (hpi) and/or 48 hpi. Gene ontology term enrichment and pathway analyses revealed that metabolic, secondary metabolite biosynthesis, plant-pathogen interaction, and plant hormone signal transduction pathways were involved. The expression of 370 cell-surface immune receptors was upregulated during infection. In addition, 188 genes encoding transcription factors were upregulated, particularly in the ERF, WRKY, bHLH, MYB, and NAC families, implying their involvement in resistance against $B$. cinerea. We further identified 325 upregulated DTGs in the hormone signal transduction pathways. Among them, the brassinosteroid (BR)-related genes were the most significantly enriched. To confirm the role of BR in Botrytis resistance, exogenous BR was applied to rose flowers before the inoculation of $B$. cinerea, which enhanced the defense response in these petals.
\end{abstract}

Conclusions: Our global transcriptome profiling provides insights into the complex gene regulatory networks mediating the rose petal response to $B$. cinerea. We further demonstrated the role of the phytohormone BR in the resistance of petals to necrotrophic fungal pathogens.

Keywords: Rose, Botrytis cinerea, Transcriptome, Cell surface receptors, Transcription factors, Brassinosteroid, Immune response

\section{Background}

Roses (Rosa sp.) are among the most important ornamental plants, accounting for more than one-third of the total cut flower industry worldwide $[1,2]$. The global consumer market for roses is mainly localized to Europe

\footnotetext{
*Correspondence: weicong_qi@163.com; zhangzhao@cau.edu.cn

${ }^{\dagger}$ Xintong Liu and Xiaoqian Cao contributed equally to this work.

${ }^{3}$ Institute of Biotechnology, Provincial Key Laboratory of Agrobiology, Jiangsu Academy of Agricultural Sciences, Zhonglingjie 50, Nanjing 210014, China ${ }^{1}$ Beijing Key Laboratory of Development and Quality Control of Ornamental Crops, Department of Ornamental Horticulture, College of Horticulture, China Agricultural University, Yuanmingyuan Xilu 2, Beijing 100193, China Full list of author information is available at the end of the article
}

and the United States, while the major production of roses occurs in Ecuador, Kenya, and other developing countries with low labor costs and a suitable climate. For each rose flower, the transport distance from greenhouse to market therefore averages more than $1500 \mathrm{~km}$ and takes three to four days, during which time the flowers are subjected to both abiotic and biotic stresses. Gray mold disease, caused by the necrotrophic fungus Botrytis cinerea, is a major postharvest disease of roses, and can cause severe losses [3].

Plants have evolved sophisticated strategies to protect themselves from pathogen attacks. Immunity in plants is

(c) The Author(s). 2018 Open Access This article is distributed under the terms of the Creative Commons Attribution 4.0 International License (http://creativecommons.org/licenses/by/4.0/), which permits unrestricted use, distribution, and 
initially governed by cell surface immune receptors that detect pathogen-associated molecular patterns (PAMPs) or damage-associated molecular patterns (DAMPs) from the host [4]; however, only a few cell surface immune receptors have been reported to function in B. cinerea resistance. Recently, WALL-ASSOCIATED KINASE1 in Arabidopsis thaliana (AtWAK1) was shown to recognize oligogalacturonides derived from plant cell walls following their break down by fungal cell wall degradation enzymes, and activate a downstream defense response to prevent further infection by B. cinerea [5]. Moreover, RBPG1 (RESPONSIVENESS TO B. CINEREA POLYGALACTURONASES1) encodes a cell surface receptor-like protein containing extracellular leucine rich repeats, which is able to recognize endopolygalacturonases produced by $B$. cinerea; however, the overexpression of RBPG1 does not increase the sensibility of Arabidopsis to Botrytis [6]. The recognition of pathogens by plant immune receptors leads to the activation of the immune responses, which often include the reprograming of phytohormone signals, the activation of pathogen-related transcription factors (TFs), and the modification of cell walls.

Phytohormones are key components in both basal and race-specific immunity. Ethylene (ET), jasmonate (JA), salicylic acid (SA), and abscisic acid (ABA) have previously been found to play crucial roles in the defense against $\mathrm{Bo}$ trytis [7-9]. In addition, gibberellins (GAs), cytokinins (CTKs), auxin (IAA), brassinosteroids (BRs) and nitric oxide (NO) are often involved in plant immunity [7, 9]. The abundant antagonism and synergism of the phytohormones give plants a wide range of regulatory potential, enabling them to activate specific defenses in a highly efficient context [10, 11]. In Arabidopsis, the SA-dependent signaling pathway is considered to be required for defense against biotrophs, while the JA and ET pathways are important against necrotrophs [8].

TFs are also important components of plant defense, playing important roles in the coordination of hormone signal interactions, the regulation of cell wall component remodeling, and many cell physiological processes. These immunity-related TFs include members of the ethylene response factor (ERF) family [12], the WRKY DNA-binding protein (WRKY) family [13], the MYB domain protein (MYB) family [14], the TGACG motif-binding protein (TGA) family [15], the NO APICAL MERISTEM, ATAF 1, CUP-SHAPED COTYLEDONS (NAC) family [16], and the MYC family $[17,18]$. Most of the ERF and WRKY proteins participate in plant defense responses.

Here, we investigated the transcriptome dynamics of rose petals during both the early and late stages of infection by $B$. cinerea. We dissected the transcriptional network governing the rose response to $B$. cinerea infection with the aim of exploring the genetic mechanisms underpinning various aspects of this defense response, especially pathogen recognition, hormone signal transduction, and the role of regulatory TFs.

\section{Results \\ Sequencing and de novo assembly of rose petal genes following $B$. cinerea infection}

Expression profiles were obtained from rose petals following infection with $B$. cinerea. To this end, detached petal disks were obtained from the outermost whorl of rose flowers and inoculated with four $2-\mu \mathrm{L}$ drops of $B$. cinerea spore inoculum containing $10^{5}$ conidia $/ \mathrm{mL}$. The control petals were mock-inoculated using potato dextrose broth (PDB). A primary disease lesion could be observed in at least one of the four inoculum drops at $36 \mathrm{~h}$ post inoculation (hpi). By contrast, no lesions were observed on the mock-inoculated petals at $36 \mathrm{hpi}$ and $48 \mathrm{hpi}$. The time points $30 \mathrm{hpi}$ and 48 hpi were chosen for the transcriptomic analysis. The $30 \mathrm{hpi}$ time point was considered to represent the early response to $B$. cinerea infection, as it was prior to the formation of the primary disease lesions at $36 \mathrm{hpi}$. The $48 \mathrm{hpi}$ time point was investigated for the later response, when the lesions were starting to expand across the petals (Fig. 1).

The sequencing of 12 samples (three replicates each for $30 \mathrm{hpi}, 30 \mathrm{~h} \mathrm{PDB}, 48 \mathrm{hpi}$, and $48 \mathrm{~h} \mathrm{PDB}$ ) resulted in 567 million clean reads comprising a total transcript length of $136,572,005$ nt. The de novo assembly of these high-quality cleaned reads generated 55,130 clusters and 61,267 unigenes, which had an average length of $1173 \mathrm{bp}$, $\mathrm{N} 50=1755$ (Table 1). The assembled sequence length was an evaluation criterion for the quality of the assembly; 5826 of the assembled unigenes had a length greater than 3000 bp (Additional file 1: Figure S1).

To further validate the expression profiles of the RNA-Seq data, six transcripts were selected for analysis using qRT-PCR. The results from the qRT-PCR analysis were generally in agreement with the expression profiles obtained using the RNA-Seq data (Fig. 2, Additional file 2: Table S1).

\section{Dynamic transcriptome of rose petals following infection with $B$. cinerea}

Differentially transcribed genes (DTGs) between the inoculated and mock-inoculated petals were determined by comparing their transcript abundances using a cutoff ratio of $>2$ and a $p$-value $<0.5$. Compared with the control, the expression of 2707 genes were significantly changed in the inoculated petals at $30 \mathrm{hpi}$, of which 1968 were upregulated and 739 were downregulated. A total of 7658 genes were significantly differentially expressed at $48 \mathrm{hpi}$, of which 5995 were upregulated and 1663 were downregulated. We identified 2113 genes that were significantly differently expressed at both $30 \mathrm{hpi}$ and $48 \mathrm{hpi}$, and these comprised the focus of this investigation (Fig. 3).

The DTGs in the rose petals were annotated with gene ontology (GO) terms. Among all processes, genes 

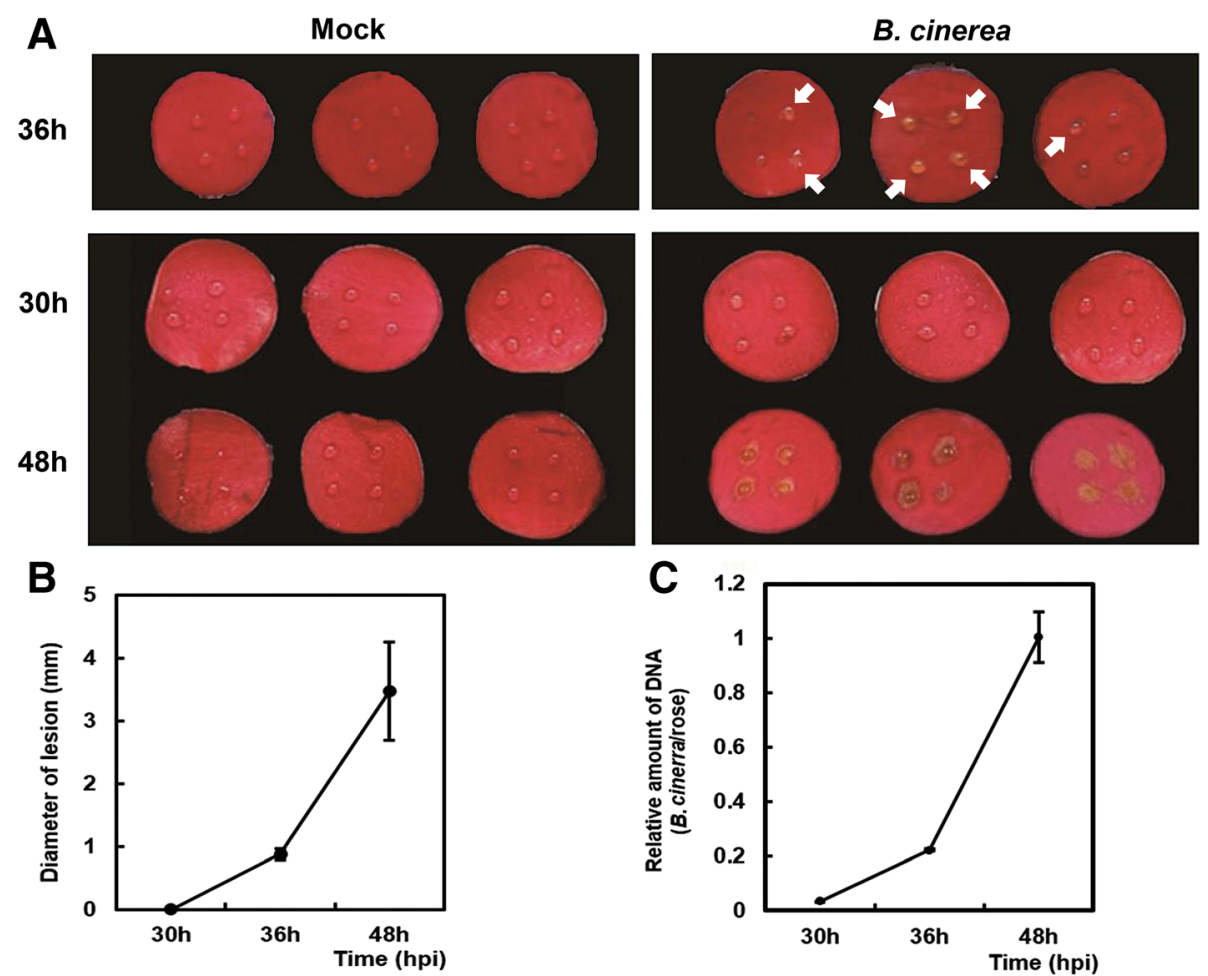

Fig. 1 B. cinerea development on rose petal disks. a Detached petal disks were obtained from the outermost whorl of rose flowers and inoculated with four $2-\mu \mathrm{L}$ drops of $B$. cinerea inoculum, containing $10^{5}$ conidia/mL, then observed at $36 \mathrm{hpi}, 30 \mathrm{hpi}$, and 48 hpi. Primary disease lesions observed at $36 \mathrm{hpi}$ were indicated by arrows. $\mathbf{b}$ Lesion diameter following inoculation. Values represent the mean diameter of 32 individual lesions, and error bars indicate SE. C) Quantification of B. cinerea biomass in rose petals. Fungal biomass was determined using qRT-PCR, comparing B. cinerea internal transcribed spacer (ITS) relative to the rose RhUbi. Error bars represent the SD of the qRT-PCR results from three independent replicates

corresponding to the metabolism, biosynthesis of secondary metabolites, plant-pathogen interactions, and plant hormone signal transduction pathways were significantly enriched at $30 \mathrm{hpi}$ and $48 \mathrm{hpi}$.

\section{Defense-regulated cell surface receptors in response to Botrytis}

Pattern recognition receptors (PRRs) located on the cell surface constitute the first line of defense for preventing the invasion of pathogens. PRRs perceive pathogens through their extracellular domains and initiate downstream disease

Table 1 Summary of the sequencing dataset used to generate the rose petal transcriptome following $B$. cinerea infection

\begin{tabular}{ll}
\hline Item & Total \\
\hline No. of reads & $567,154,672$ \\
No. of unigenes & 116,397 \\
Total length of transcripts & $136,572,005 \mathrm{nt}$ \\
Mean length of transcripts & $1173 \mathrm{bp}$ \\
No. of distinct clusters & 55,130 \\
No. of distinct singletons & 61,267 \\
N50 & 1755 \\
\hline
\end{tabular}

responses through intracellular domains, co-receptors, or intracellular binding proteins that bind to them for signal transmission $[6,19,20]$. Based on annotations using the Non-redundant Protein Sequence (NR), GO, and Kyoto Encyclopedia of Genes and Genomes (KEGG) databases, 370 cell-wall receptor proteins were among the differentially expressed genes, including 38 wall-associated receptor kinases (WAKs), 138 leucine-rich repeat receptors (LRRs), 47 cysteine-rich receptor-like protein kinases (CRKs), 11 lysM-domain receptor kinases (LYKs), and 136 lectin-domain containing receptor kinases (LecRKs) (Fig. 4a, Additional file 3: Table S2).

\section{Defense-induced hormone signal transduction in rose petals}

Hormones act as internal cues to initiate plant defenses. We identified 325 upregulated genes related to hormone signal transduction pathways in the DTGs, according to the pathway ID and KO definitions. More specifically, $54.5 \%$ (177/325) were associated with BR signaling, $11.1 \%$ (36) with GA, 9.8\% (32) with ET, 7.4\% (24) with ABA, 5.5\% (18) with IAA, 5.5\% (18) with JA, 4.0\% (13) with SA, and $2.2 \%$ (7) with CTK signaling (Fig. 4b, Additional file 4: Table S3). Specifically, a simplified 


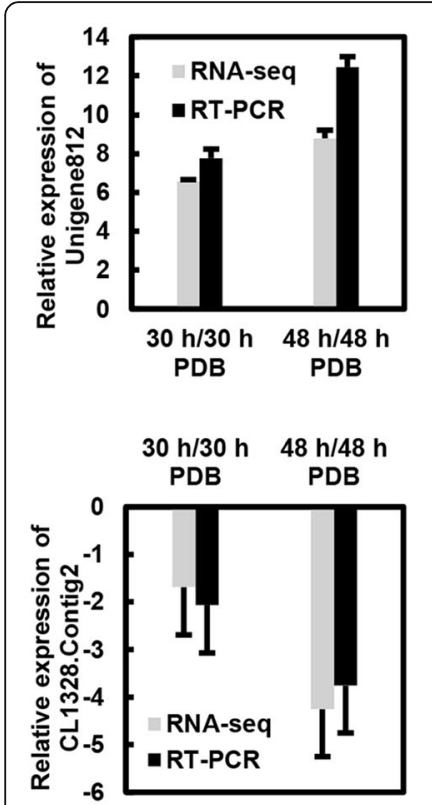

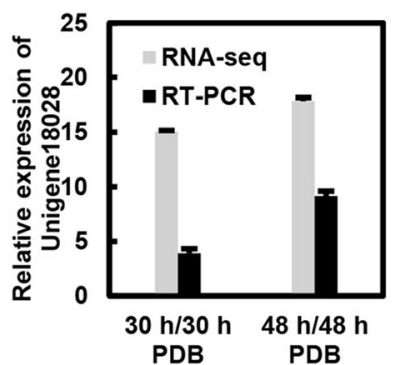
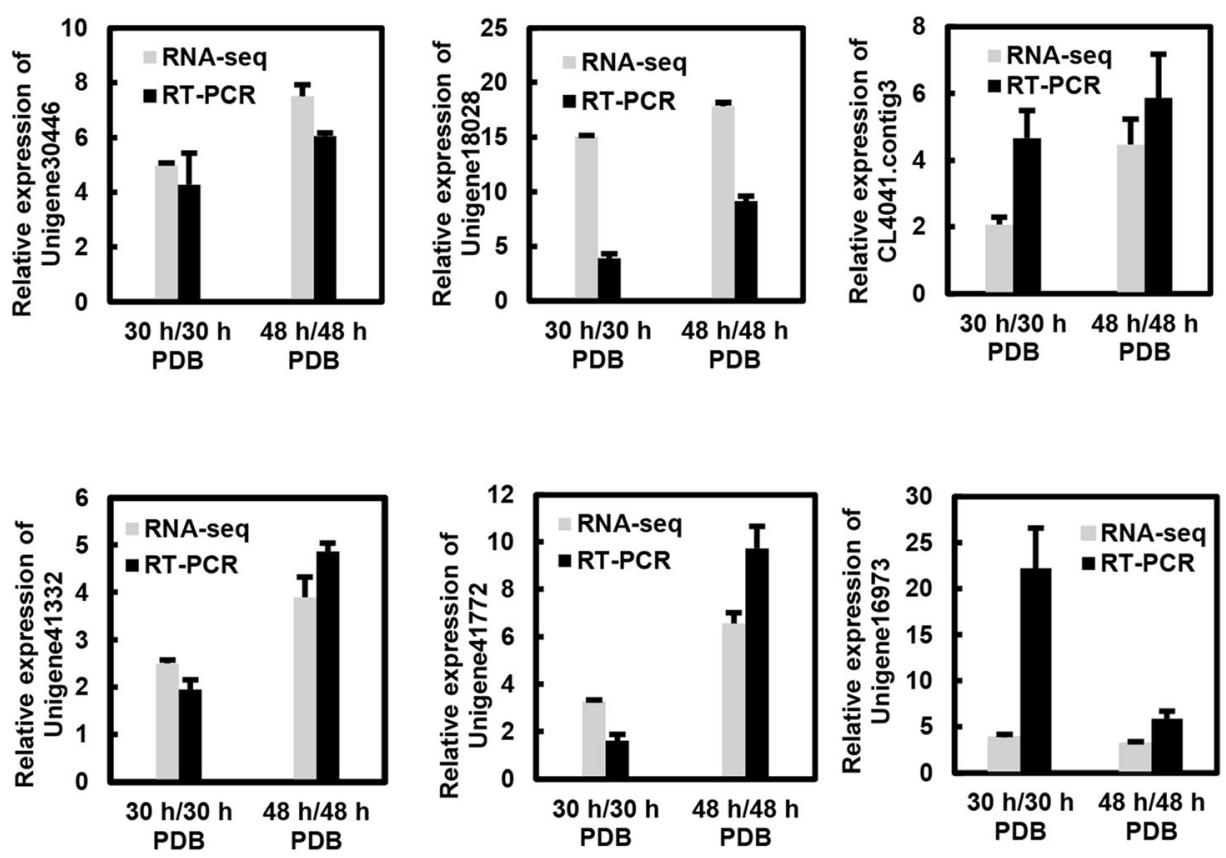

Fig. 2 Validation of RNA-Seq results using qRT-PCR. PDB; potato dextrose broth; Unigene812, allene oxide synthase-like 3; Unigene30446, pleiotropic drug resistance protein 3-like; Unigene18028, uncharacterized protein; CL1328.Contig2, BTB/POZ domain-containing protein;

Unigene41332, wall-associated receptor kinase-like 1; Unigene41772, WEB family protein; CL4041.contig3, interleukin-1 receptor-associated kinase 4; Unigene16973, brassinosteroid insensitive 1-associated receptor kinase 1. RhUbi was used as an internal control. Values are the means of three biological replicates \pm SD

brassinosteroid signaling pathway showed that $49 B A K 1$, 21 BRI1, one BSK, and two BIN2 genes were significantly upregulated (Fig. 4d). ET and JA signaling have previously been reported to play an important role in disease defense, and several genes involved in these signaling pathways were found to be upregulated during $B$. cinerea infection (Fig. 4d). In the ET signal transduction pathway, we identified three $E T R$, five $C T R 1$, and 45 ET-RESPONSIVE TF (ERF) DTGs. In the JA signal transduction pathway, we found $10 \mathrm{JAZ}$ and $12 \mathrm{MYC} 2 /$ $b H L H$ family TFs that were differentially expressed during $B$. cinerea infection.
Defense-responsive TFs in rose petals

Of the DTGs, we identified 188 TFs that were upregulated in rose petals following the $B$. cinerea inoculation, including ERF, WRKY, bHLH, MYB, NAC, bZIP, TGA, HSF, GTE, MADS-box, MYC, trihelix, zinc-finger, and NFYC family members (Fig. 4c). Among the nine TF families involved, the most commonly observed was the ERF family, with 46 members that had a differential expression ratio far greater than 2 (Additional file 5: Table S4). The upregulated TFs were sensitive to $B$. cinerea, and many were regulated by hormonal signals, especially BR, ET, and JA (Fig. 4d).
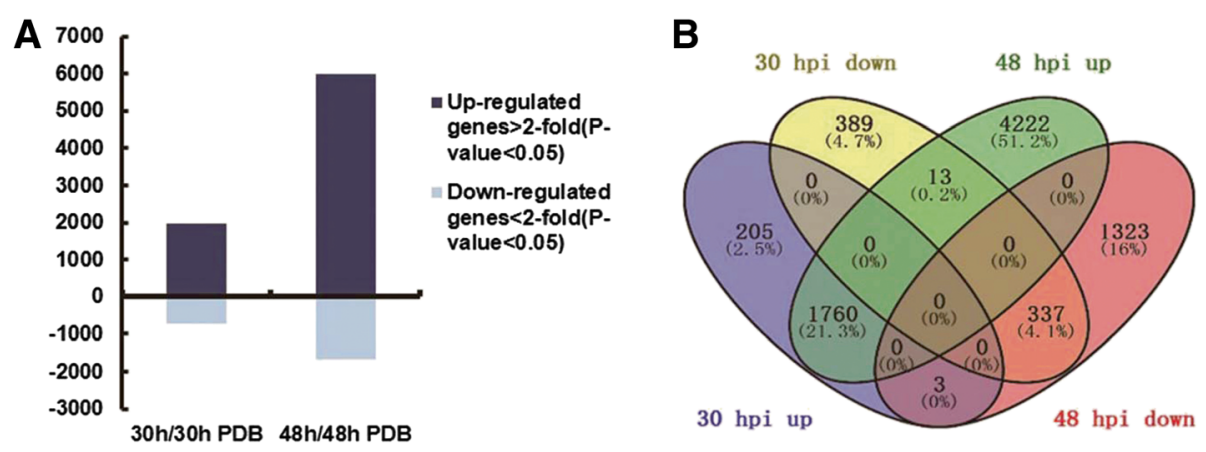

Fig. 3 Numbers of DTGs between inoculated and control rose petals at 30 hpi and 48 hpi. a Numbers of DTGs in petals. b Wayne figure of 30 hpi and 48 hpi. Up represents upregulated genes, down represents downregulated genes 


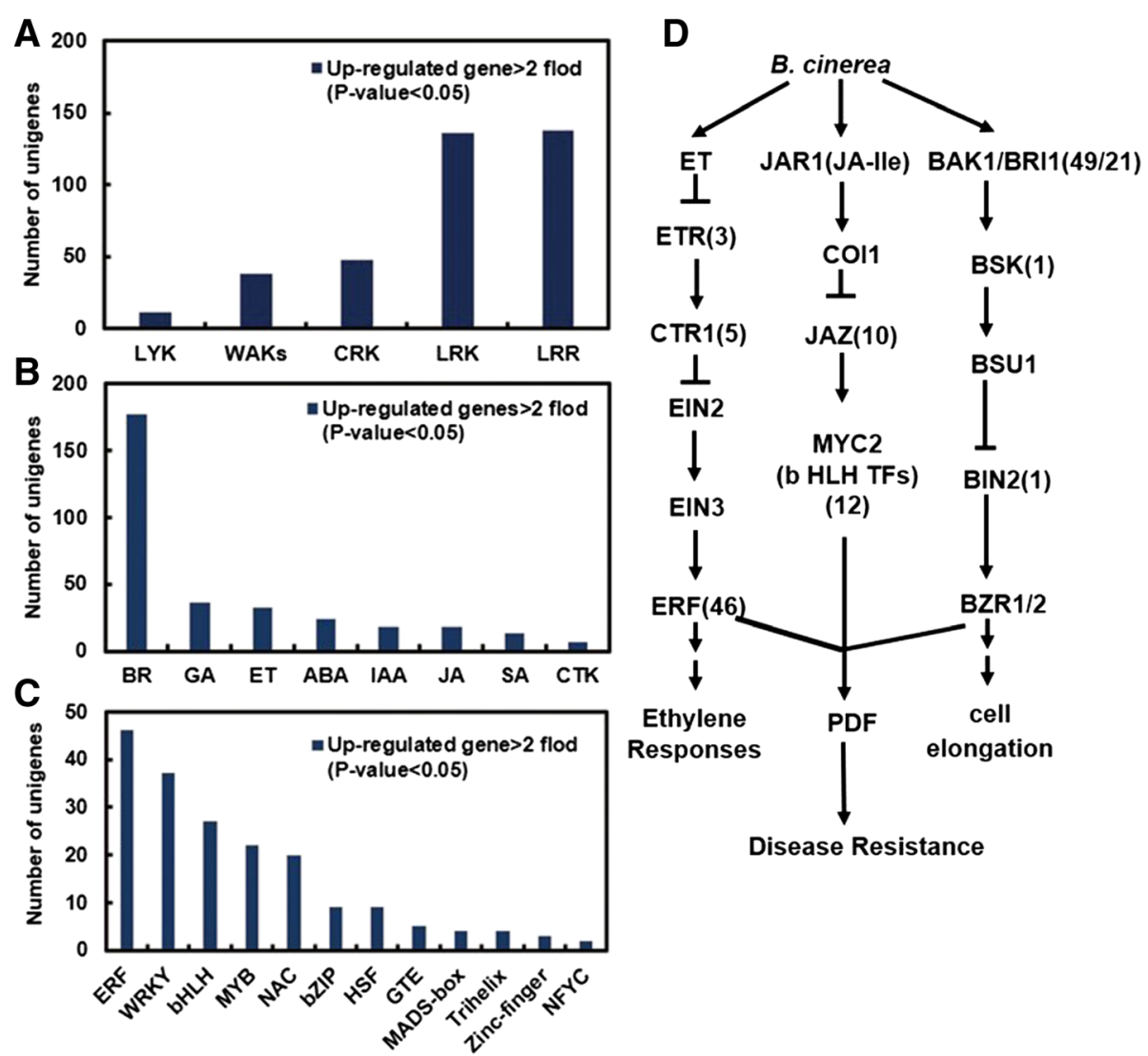

Fig. 4 Distribution of cell-wall receptor proteins, hormone signal transcripts, and TF-related DTGs during petal infection. DTGs were determined using a cutoff ratio of $>2$ ( $p$-value $<0.05$ ), when comparing expression at $30 \mathrm{hpi}$ and $48 \mathrm{hpi}$ with that of the control. a Number of unigenes corresponding to cell-wall receptors. LYK: lys domain receptor; WAKs: wall-associated receptor kinase; CRKs, cysteine-rich receptor-like protein kinases; LecRK: lectin receptor kinase; LRR: leucine repeat receptor. $\mathbf{b}$ Number of hormone signal-related DTGs in infected rose petals. BR: brassinosteroid; GA: gibberellin; ABA: abscisic acid; ET: ethylene; IAA: auxin; JA: jasmonate; CTK: cytokinin; SA: salicylic acid. c Number of TF DTGs in infected rose petals. $\mathbf{d}$ Simplified ET and JA signal transduction induced by B. cinerea infection [52]. CTR1: constitutive triple response; EIN2: ethylene insensitive 2; EIN3: ethylene insensitive 3; ERF: ethylene response factor; JA-lle: jasmonate-isoleucine; SCF: Skp/Cullin/F-box; COl1: coronatine-insensitive 1; JAZ: jasmonate ZIM domain; bHLH TFs: basic helix-loop-helix TFs; BAK1/BRI1, brassinosteroid insensitive 1-associated receptor kinase 1; BSK1, BR-signaling kinase; BSU1, serine/threonine-protein phosphatase; BIN2, protein brassinosteroid insensitive 2; BZR1/2, brassinosteroid resistant 1/2; PDF1.2: plant defensin 1.2. The numbers in parentheses correspond to the number of upregulated unigenes

\section{Exogenous application of BR promotes rose petal}

\section{resistance against $B$. cinerea}

Among the eight hormone signal transduction pathways induced by $B$. cinerea infection, the BR signaling pathway was represented in the DTGs to a far greater extent than the others. Nevertheless, the role of BR in plant defense against $B$. cinerea is largely unknown. To confirm the crucial role of BR in the petal defense mechanism, we treated rose flowers with $5 \mathrm{mM} \mathrm{BR}$, then inoculated them with $B$. cinerea (Fig. 5a). The BR treatment significantly decreased the diameter of the lesions that formed at the inoculation sites (Fig. 5b), suggesting that BRs might play an important role in the resistance to $B$. cinerea in rose petals.

\section{BR INSENSITIVE 1-ASSOCIATED RECEPTOR KINASE 1 is} required for $B$. cinerea resistance BR INSENSITIVE 1-ASSOCIATED RECEPTOR KINASE 1 (BAK1) is a protein that interacts with the BR receptor BRI1, and play a critical role in BR signaling [21, 22]. To investigate whether $B A K 1$ contributes to $B$. cinerea resistance, three independent Arabidopsis mutants, bak1-3 (Salk_034523) [23], bak1-4 (Salk_116202) [23] and bak1-5 (C408Y in 10th exon) [24] were inoculated with B. cinerea (Additional file 6: Figures S2A). Disease development was quantified by measuring the size of the lesions at $72 \mathrm{~h}$ post inoculation. Compared with wild type plant, all three mutants showed significantly enlarged diameter of the lesions (Additional file 6: 


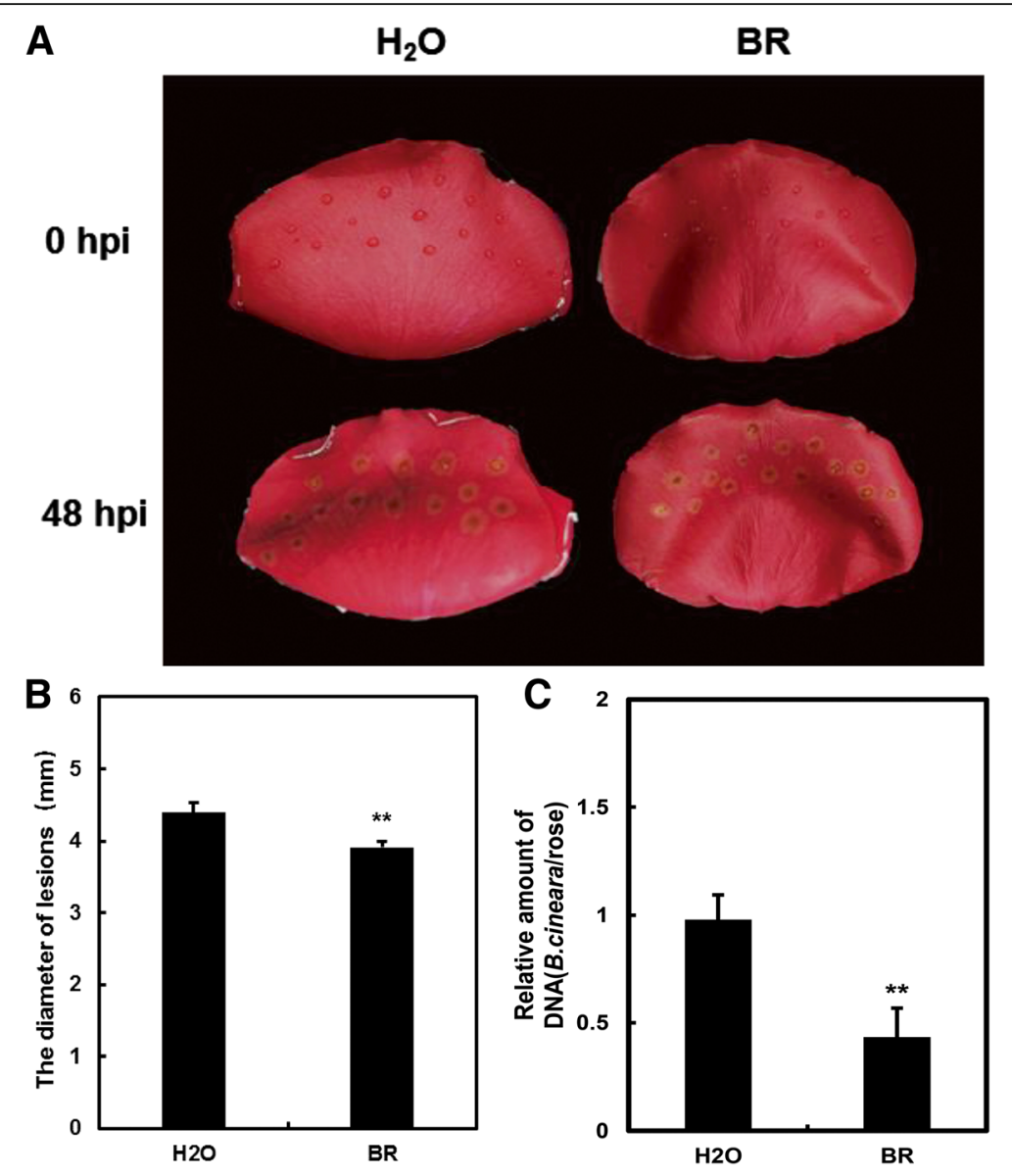

Fig. 5 Exogenous BR promotes rose petal resistance to B. cinerea. a Mock- and BR-treated rose petals inoculated with $B$. cinerea. $\mathbf{b}$ The diameter of the lesions in petals subjected to exogenous BR or water. Values are the mean of four biological replicates using 16 rose petals (Student's t-test, $p$-value $<0.01)$. c) Quantification of $B$. cinerea biomass in rose petals. Fungal biomass was determined using qRT-PCR, comparing B. cinerea internal transcribed spacer (ITS) relative to the rose RhUbi. Error bars represent the SD of the GRT-PCR results from three independent replicates

Fig. S2B), suggesting that BAK1 is required for plant resistance against $B$. cinerea.

\section{Discussion}

Despite the important economic impact of gray mold disease on roses, the details of rose defenses during infection by $B$. cinerea remain largely unknown. Our identification of DTGs in rose petals during the early stages of infection provides an overview of the mechanisms that might be involved in their defense response. We identified 370 cell-wall receptor protein unigenes, 325 hormone signal transduction pathway-related unigenes, and 188 TF unigenes that were upregulated following $B$. cinerea inoculation, which are likely to mediate the rose immune response from recognition to defense.

Plants use several types of cell surface receptors to perceive extracellular signals, such as PAMPs. In this study, five categories of cell-wall receptor protein were found to be involved in the rose petal response to $B$. cinerea. The first category, the LRRs, contains the conserved leucine repeat sequences, which bind proteins and polypeptides such as fungal endopolygalacturonases [25]. Recently, an Arabidopsis LRR, RBPG1, was reported to recognize fungal endopolygalacturonases from $B$. cinerea, lending support to our findings. Another cell-wall receptor protein category, the carbohydrate-binding LecRKs, were also found to be involved in the rose petal defense response. In Arabidopsis, lecrk-VI.2-1 mutants were less able to upregulate the expression of the pattern-triggered immunity (PTI) marker genes, while plants overexpressing LecRK-VI.2 had an increased PTI response, demonstrating that LecRK-VI.2 is a novel mediator of the Arabidopsis PTI response [26]. We found that the CRKs were also involved in the rose petal response to $B$. cinerea. These receptors are characterized by the presence of one to four copies of domain 26 of unknown function (duf26), a C-X8-C-X2-C motif in the extracellular receptor region, in their $\mathrm{N}$-terminus and a serine/threonine kinase domain in their C-terminus [27]. In barley (Hordeum vulgare), the transient silencing of $H \nu C R K 1$ expression in bombarded epidermal cells led to 
an enhanced resistance to Blumeria graminis, but did not affect $R$-gene-mediated resistance [28]. Another cell-wall receptor category identified in our study, the LYKs, were previously demonstrated to be essential for chitin signaling (likely as a part of the receptor complex) and the induction of plant innate immunity $[29,30]$. The fifth category of cell-wall receptors involved in the rose petal response to $B$. cinerea, the WAKs, are similar to epidermal growth factors and can bind oligonated galacturonic acid glycosides produced by the degradation of the plant cell wall itself, activating downstream immune responses to prevent further infection by the fungus [31, 32]. Unlike the first four receptor types, the WAK receptors do not directly perceive the pathogen itself, but rather recognize its effects. These cell-wall receptors, which were upregulated in rose petals during early infection, may provide evidence of the $B$. cinerea recognition mechanisms in rose.

The crosstalk and fine-tuning of the hormone signal transduction networks in plant immunity are a major focus for current research. Plant hormones such as SA, JA, and ET play a vital role in signaling the presence of infection and initiating the downstream defense responses. In our study, the number of unigenes corresponding to the JA and SA signaling pathways was relatively low, possibly because the wounding response initiated when the petal disks were cut may have masked the changes of these pathways in response to the gray mold infection. The expression of BR-related genes was significantly enriched in the infected petals, suggesting their potential role in the petal defense response. BRs can act antagonistically or synergistically with responses to PAMPs, and the synergistic activities of BRs on PAMP responses are known to require BAK1 [33]. After infection with the pathogen, the expression of ET biosynthesis genes also increased. ET can induce the activation and accumulation of pathogenesis-related proteins and antimicrobial peptides, including glucanase, chitinase, and osmotin.

We identified DTGs encoding TFs in the ERF family, the WRKY family, the bHLH family, the MYB family, and the NAC family. ERF-family TFs integrate and communicate signals involving SA, JA, and ET [17]. In Arabidopsis, ERF1, ERF5, ERF6, RAP2.2 (related to AP2.21), and ORA59 (octadecanoid-responsive Arabidopsis AP2/ ERF59) are involved in the regulation of plant defense against $B$. cinerea $[12,34-37]$. The WRKY family participates in the responses to a variety of abiotic and biological stresses. WRKY33 was reported to be upregulated by a variety of defense response pathways following $B$. cinerea infection [13]. The TFs of the MYB and NAC families are involved in ABA-JA interactions and serve as important regulators of the plant responses to abiotic and biological stresses $[38,39]$. ABA can promote susceptibility to gray mold and reduce the expression of JA/ET defense-related genes by influencing the activities of the ERF1 and
ORA59 TFs in the ET signaling pathway [40-42]. MYC2 is thought to be a positive regulator of the ABA signaling pathway and a key factor mediated by $\mathrm{JA}$, and is known to antagonize the regulation of the JA response to dead parasites [43]. These TFs are regulated by the phytohormones to control the expression of the downstream defense-related genes and enhance plant immunity.

\section{Conclusions}

In conclusion, the present research, focusing on the grey mold infection of rose petals, provides a large amount of relevant transcriptomic information, from which a genetic defense-response network was elucidated. The results suggest that the phytohormone BR plays a critical role in rose petal defense against $B$. cinerea. Further study on this phytohormone and related DTGs can provide us novel insights into rose resistance to $B$. cinerea and this knowledge can be exploited for durable resistance against this pathogen.

\section{Methods \\ Plant and fungal growth and plant infection}

Roses (Rosa hybrida, cv. Samantha) were grown in glasshouses in Nankou, Changping District, Beijing, China. Rose flowers with fully open buds were harvested at developmental stage 2 , their stems were immediately placed in water. The flower stems were re-cut to $20 \mathrm{~cm}$ in length under water and placed in deionized water to await further processing. The rose petals were cut into $12.5-\mathrm{mm}$ disks, which were placed on $0.4 \%$ water agar, with 16 disks per petri dish.

The $B$. cinerea inoculum was produced by growing strain B05.10 [44] on a solid medium (potato dextrose agar; $39 \mathrm{~g}$ per $\mathrm{L} \mathrm{dH} 2 \mathrm{O}, \mathrm{pH} \approx 5.6$ ) at room temperature for 14 days. Spore inoculum was prepared by harvesting spores in water, filtering through glass wool to remove the hyphae, and suspending the filtrate in potato dextrose broth (PDB; $24 \mathrm{~g}$ per $\mathrm{L} \mathrm{dH} 2 \mathrm{O}$ ) with $10^{5}$ conidia/mL. Four $2-\mu \mathrm{L}$ drops of B. cinerea inoculum or PDB (mock) were dropped onto each petal disk. Infected and control disks were individually sampled in a randomized manner from each of the three trays at $30 \mathrm{hpi}$ and $48 \mathrm{hpi}$, with three biological repeats for both infected and control treatments at each time point. Petal disks were immediately frozen in liquid nitrogen at the time of harvesting and stored at $-80{ }^{\circ} \mathrm{C}$.

Arabidopsis plants were grown in a climate chamber at $22{ }^{\circ} \mathrm{C}$ and $70 \%$ relative humidity under a $16 / 8 \mathrm{~h}$ light/ dark cycle. Leaves of 25 days old Arabidopsis plants were inoculated with $2-\mu \mathrm{L}$ drops of $B$. cinerea inoculum. Finally, 6-7 leaves per Arabidopsis plant and 4 plants per genotype were used for inoculation, leading to a total of at least 24 lesions per mutant. Lesion sizes were 
measured at $72 \mathrm{hpi}$ and analyzed statistically by a Student's $t$-test.

\section{Total RNA extraction and RNA-Seq library preparation}

The material for RNA-seq are petal discs, as showed in Fig. 1a. Total RNA was extracted using the hot borate method as previously described [45], and treated with RNase-free DNase I (Promega) to remove any contaminating genomic DNA. Three biological repeats were performed for both time points. Strand-specific RNA libraries were constructed using the protocol described previously [46], then sequenced on a HiSeq 2500 system (Illumina), according to the manufacturer's instructions (Additional file 7). The raw reads were deposited into the NCBI SRA database under accession no. PRJNA414570.

\section{RNA-Seq data processing, assembly, and annotation}

First, the raw data was cleaned by removing the adaptor-containing sequences,poly-N, and low-quality reads, then reads shorter than $40 \mathrm{bp}$ were removed with Q-value $\leq 5$. The remaining high-quality, clean reads were used in subsequent analyses. The remaining high-quality, clean reads were used in subsequent analyses. Assembly was performed with Trinity software [47] with min_kmer_cov set to generate contigs and unigenes. All other parameters set to their defaults. To remove the redundancy of the Trinity-assembled contigs, the contigs were again assembled de novo using iAssembler [44-48] (Additional file 8). The final unigenes were annotated using the NR (NCBI non-redundant protein), NT (NCBI non-redundant transcript), Swiss-Prot, KEGG (KEGG Ortholog), KOG (eukaryotic Ortholog Groups), GO libraries.Using the BLASTX algorithm with a significance threshold of E-value $\leq 10^{-5}$. The unigene expression was calculated using the FPKM (fragments per kb per Million reads) method. DTGs were analyzed by the edge $R \mathrm{R}$ package and defined as genes with a false discovery rate of $<0.001$ and at least a two-fold difference. Transcription factors were predicted by BLASTX searching of plantTFDB with E-value $\leq 10^{-5}$. KEGG pathway enrichment of DTGs was performed using KOBAS. The GO term enrichment was analysis by the GOseq $\mathrm{R}$ package based on Wallenius non-central hyper-geometric distribution.

\section{Quantitative RT-PCR}

To confirm the RNA-Seq results, the transcript abundance of six selected genes was analyzed using qRT-PCR, as previously described [49]. Briefly, the total RNAs of three biological repeats were equivalently mixed for each sample. cDNA was generated using Takara Reverse Transcriptase M-MLV, and $1 \mu \mathrm{L}$ of the first strand cDNA was used as a template in the reaction with the KAPATM SYBRR quantitative PCR kit (Takara), which was run on a
StepOnePlus Real-Time PCR System (Thermo Fisher Scientific). RhUbi was used as a housekeeping gene [50]. The primers used for determining transcript abundance are listed in Additional file 2: Table S1.

\section{Exogenous BR treatment}

Flowering rose stems were cut to lengths of $25 \mathrm{~cm}$ and placed in $5 \mu \mathrm{M}$ 2,4-epibrassinolide [51] for $24 \mathrm{~h}$, with two stems per bottle. Stems treated with water were used as the control. The BR-treated and control petals were inoculated with $2 \mu \mathrm{L}$ B. cinerea spore inoculum and kept on $0.4 \%$ water agar, as described above. The diameters of the lesions were measured at $48 \mathrm{hpi}$.

\section{Additional files}

Additional file 1: Figure S1. Length distribution of the assembled unigenes. (DOCX $37 \mathrm{~kb}$ )

Additional file 2: Table S1. List of all primers used in this study. (DOCX $15 \mathrm{~kb}$ )

Additional file 3: Supplementary Table S2. Defense-regulated cell surface receptors in response to Botrytis. (XLSX $47 \mathrm{~kb}$ )

Additional file 4: Supplementary Table S3. Defense-induced hormone signal related genes in rose petals. (XLSX $26 \mathrm{~kb}$ )

Additional file 5: Supplementary Table S4. Defense-regulated transcriptional factors in response to Botrytis. (XLSX $35 \mathrm{~kb}$ )

Additional file 6: Figure S2. BR INSENSITIVE 1-ASSOCIATED RECEPTOR KINASE 1 is required for $B$. cinerea resistance. A) The schematic of the genomic structure of BAK1. Exons and introns are indicated with black boxes and lines, respectively. The T-DNA insertion sites are marked with triangles and missense mutation sites are indicated with arrows. B) All three independent mutant alleles of BAK1 used in this study showed compromised resistance against B. cinerea. (DOCX 146 kb)

Additional file 7: Table S5. All sample clean data statistics. (DOCX $14 \mathrm{~kb}$ ) Additional file 8: Supplementary Data. All assembled Unigenes. (FA $125981 \mathrm{~kb})$

\section{Abbreviations}

ABA: abscisic acid; BRs: brassinosteroids; CRKs: cysteine-rich receptor-like protein kinases; CTKs: cytokinins; DAMPs: damage-associated molecular patterns; DTGs: differentially transcribed genes; ET: ethylene; GAs: gibberellins; hpi: hours post inoculation; IAA: auxin; JA: jasmonate; LecRKs: lectin-domain containing receptor kinases; LRRs: leucine-rich repeat receptors; LYKs: lysMdomain receptor kinases; NO: nitric oxide; PAMPs: pathogen-associated molecular patterns; PDB: potato dextrose broth; PRRs: pattern recognition receptors; SA: salicylic acid; TFs: transcription factors; WAKs: wall-associated receptor kinases

\section{Funding}

Zhao Zhang is supported by the National Natural Science Foundation of China (31501791 and 31772344). This research is funded in part by the Natural Science Foundation of Beijing Municipality (6162017 to Zhao Zhang). The funders played no role in study design, data collection and analysis, decision to publish, or preparation of the manuscript.

\section{Availability of data and materials}

Raw data supporting our findings can be found in the National Center for Biotechnology Information (NCBI) database under the accession number PRJNA414570

\section{Authors' contributions}

$W Q, X C$, and $Z Z$ conceived the research and designed the experiments. $X C$ $X L, S S, N Z, D L$, and PF performed the experiments; SS and DL provided 
technical support and analyzed the data; WQ and XC drafted the manuscript; and WQ and ZZ revised the manuscript. All the authors have read and approved the final version of the manuscript.

\section{Ethics approval and consent to participate}

Not applicable. Our research did not involve any human or animal subjects, material, or data. The plant materials used in this study were provided by the China Agricultural University and are freely available for research purposes following institutional, national and international guidelines.

\section{Consent for publication}

Not applicable.

\section{Competing interests}

The authors declare that they have no competing interests.

\section{Publisher's Note}

Springer Nature remains neutral with regard to jurisdictional claims in published maps and institutional affiliations.

\section{Author details}

${ }^{1}$ Beijing Key Laboratory of Development and Quality Control of Ornamental Crops, Department of Ornamental Horticulture, College of Horticulture, China Agricultural University, Yuanmingyuan Xilu 2, Beijing 100193, China. ${ }^{2}$ Institute of Plant Protection, Jiangsu Academy of Agricultural Sciences, Nanjing, China. ${ }^{3}$ Institute of Biotechnology, Provincial Key Laboratory of Agrobiology, Jiangsu Academy of Agricultural Sciences, Zhonglingjie 50, Nanjing 210014, China

Received: 9 November 2017 Accepted: 10 August 2018 Published online: 20 August 2018

\section{References}

1. Koning-Boucoiran CF, Esselink GD, Vukosavljev M, van't Westende WP, Gitonga WW, Krens FA, Voorrips RE, van de Weg WE, Schulz D, Debener T. Using RNA-Seq to assemble a rose transcriptome with more than 13,000 full-length expressed genes and to develop the WagRhSNP 68k Axiom SNP array for rose (Rosa L.). Front Plant Sci. 2015;6:249.

2. Liorzou M, Pernet A, Li S, Chastellier A, Thouroude T, Michel G, Malécot V, Gaillard S, Briée C, Foucher F. Nineteenth century French rose (Rosa sp.) germplasm shows a shift over time from a European to an Asian genetic background. J Exp Bot. 2016;67(15):4711-25.

3. Dean R, Van Kan JA, Pretorius ZA, Hammond-Kosack KE, Di Pietro A, Spanu PD, Rudd JJ, Dickman M, Kahmann R, Ellis J. The top 10 fungal pathogens in molecular plant pathology. Mol Plant Pathol. 2012;13(4):414-30.

4. Jones JD, Dangl JL. The plant immune system. Nature. 2006;444(7117):323-9.

5. Brutus A, Sicilia F, Macone A, Cervone F, De Lorenzo G. A domain swap approach reveals a role of the plant wall-associated kinase 1 (WAK1) as a receptor of oligogalacturonides. Proc Natl Acad Sci. 2010;107(20):9452-7.

6. Zhang Z, Song Y, Liu C-M, Thomma BP. Mutational analysis of the Ve immune receptor that mediates Verticillium resistance in tomato. PLoS One. 2014;9(6):e99511.

7. Audenaert K, De Meyer GB, Höfte MM. Abscisic acid determines basal susceptibility of tomato toBotrytis cinerea and suppresses salicylic aciddependent signaling mechanisms. Plant Physiol. 2002;128(2):491-501.

8. Glazebrook J. Contrasting mechanisms of defense against biotrophic and necrotrophic pathogens. Annu Rev Phytopathol. 2005:43:205-27.

9. Belkhadir Y, Jaillais Y, Epple P, Balsemão-Pires E, Dangl JL, Chory J. Brassinosteroids modulate the efficiency of plant immune responses to microbe-associated molecular patterns. Proc Natl Acad Sci. 2012;109(1):297-302.

10. Pieterse CM, Van der Does D, Zamioudis C, Leon-Reyes A, Van Wees SC Hormonal modulation of plant immunity. Annu Rev Cell Dev Biol. 2012;28: 489-521.

11. Vos I, Pieterse C, Wees S. Costs and benefits of hormone-regulated plant defences. Plant Pathol. 2013;62(S1):43-55.

12. Berrocal-Lobo M, Molina A, Solano R. Constitutive expression of ETHYLENERESPONSE-FACTOR1 in Arabidopsis confers resistance to several necrotrophic fungi. Plant J. 2002;29(1):23-32

13. Zheng Z, Qamar SA, Chen Z, Mengiste T. Arabidopsis WRKY33 transcription factor is required for resistance to necrotrophic fungal pathogens. Plant $\mathrm{J}$. 2006;48(4):592-605.
14. Mengiste T, Chen X, Salmeron J, Dietrich R. The BOTRYTIS SUSCEPTIBLE1 gene encodes an R2R3MYB transcription factor protein that is required for biotic and abiotic stress responses in Arabidopsis. Plant Cell. 2003;15(11): 2551-65.

15. Ou B, Yin K-Q, Liu S-N, Yang Y, Gu T, Hui JMW, Zhang L, Miao J, Kondou Y, Matsui M. A high-throughput screening system for Arabidopsis transcription factors and its application to Med25-dependent transcriptional regulation. Mol Plant. 2011;4(3):546-55.

16. Le Hénanff G, Profizi C, Courteaux B, Rabenoelina F, Gérard C, Clément C, Baillieul F, Cordelier S, Dhondt-Cordelier S. Grapevine NAC1 transcription factor as a convergent node in developmental processes, abiotic stresses, and necrotrophic/biotrophic pathogen tolerance. J Exp Bot. 2013;64(16): 4877-93.

17. Lorenzo O, Chico JM, Sánchez-Serrano JJ, Solano R. JASMONATEINSENSITIVE1 encodes a MYC transcription factor essential to discriminate between different jasmonate-regulated defense responses in Arabidopsis. Plant Cell. 2004;16(7):1938-50.

18. Du M, Zhao J, Tzeng DT, Liu Y, Deng L, Yang T, Zhai Q, Wu F, Huang Z, Zhou M. MYC2 Orchestrates a Hierarchical Transcriptional Cascade that Regulates Jasmonate-Mediated Plant Immunity in Tomato. The Plant Cell Online. 2017; tpc. 00953.02016

19. Zhang Z, Thomma BP. Virus-induced gene silencing and agrobacterium tumefaciens-mediated transient expression in Nicotiana tabacum. PlantPathog Interact: Methods Protoc. 2014:173-81.

20. Fradin EF, Zhang Z, Rovenich H, Song Y, Liebrand TW, Masini L, van den Berg GC, Joosten MH, Thomma BP. Functional analysis of the tomato immune receptor Ve1 through domain swaps with its non-functional homolog Ve2. PLoS One. 2014;9(2):e88208.

21. Li J, Wen JQ, Lease KA, Doke JT, Tax FE, Walker JC. BAK1, an Arabidopsis LRR receptor-like protein kinase, interacts with BRI1 and modulates brassinosteroid signaling. Cell. 2002;110(2):213-22.

22. Nam KH, Li JM. BRI1/BAK1, a receptor kinase pair mediating brassinosteroid signaling. Cell. 2002;110(2):203-12.

23. Chinchilla D, Bauer Z, Regenass M, Boller T, Felix G. The Arabidopsis receptor kinase FLS2 binds flg22 and determines the specificity of flagellin perception. Plant Cell. 2006;18(2):465-76.

24. Schwessinger B, Ronald PC. Plant innate immunity: perception of conserved microbial signatures. Annu Rev Plant Biol. 2012;63:451-82.

25. Takai R, Isogai A, Takayama S, Che F-S. Analysis of flagellin perception mediated by flg22 receptor OsFLS2 in rice. Mol Plant-Microbe Interact. 2008; 21(12):1635-42.

26. Singh P, Kuo Y-C, Mishra S, Tsai C-H, Chien C-C, Chen C-W, DesclosTheveniau M, Chu P-W, Schulze B, Chinchilla D. The lectin receptor kinase-VI. 2 is required for priming and positively regulates Arabidopsis patterntriggered immunity. Plant Cell. 2012;24(3):1256-70.

27. Shiu S-H, Karlowski WM, Pan R, Tzeng Y-H, Mayer KF, Li W-H. Comparative analysis of the receptor-like kinase family in Arabidopsis and rice. Plant Cell. 2004;16(5):1220-34.

28. Rayapuram C, Jensen MK, Maiser F, Shanir JV, Hornshøj H, Rung JH, Gregersen PL, Schweizer P, Collinge DB, Lyngkjær MF. Regulation of basal resistance by a powdery mildew-induced cysteine-rich receptor-like protein kinase in barley. Mol Plant Pathol. 2012;13(2):135-47.

29. Wan J, Zhang X-C, Neece D, Ramonell KM, Clough S, Sy K, Stacey MG, Stacey G. A LysM receptor-like kinase plays a critical role in chitin signaling and fungal resistance in Arabidopsis. Plant Cell. 2008;20(2):471-81.

30. Miya A, Albert P, Shinya T, Desaki Y, Ichimura K, Shirasu K, Narusaka Y, Kawakami $\mathrm{N}$, Kaku H, Shibuya N. CERK1, a LysM receptor kinase, is essential for chitin elicitor signaling in Arabidopsis. Proc Natl Acad Sci. 2007;104(49):19613-8.

31. Wirthmueller L, Maqbool A, Banfield MJ. On the front line: structural insights into plant-pathogen interactions. Nat Rev Microbiol. 2013;11(11):761-76.

32. Kohorn BD. The state of cell wall pectin monitored by wall associated kinases: a model. Plant Signal Behav. 2015;10(7):e1035854.

33. Belkhadir $Y$, Jaillais $Y$, Epple $P$, Balsemao-Pires E, Dangl JL, Chory J. Brassinosteroids modulate the efficiency of plant immune responses to microbe-associated molecular patterns. P Natl Acad Sci USA. 2012;109(1):297-302

34. Moffat CS, Ingle RA, Wathugala DL, Saunders NJ, Knight H, Knight MR. ERF5 and ERF6 play redundant roles as positive regulators of JA/etmediated defense against Botrytis cinerea in Arabidopsis. PLoS One. 2012;7(4):e35995

35. Son GH, Wan J, Kim HJ, Nguyen XC, Chung WS, Hong JC, Stacey G. Ethylene-responsive element-binding factor 5, ERF5, is involved in chitin- 
induced innate immunity response. Mol Plant-Microbe Interact. 2012;25(1): 48-60.

36. Meng $X, X u J, H e ~ Y$, Yang K-Y, Mordorski B, Liu Y, Zhang S. Phosphorylation of an ERF transcription factor by Arabidopsis MPK3/MPK6 regulates plant defense gene induction and fungal resistance. Plant Cell. 2013;25(3):1126-42.

37. Wehner N, Hartmann L, Ehlert A, Böttner S, Oñate-Sánchez L, Dröge-Laser W. High-throughput protoplast transactivation (PTA) system for the analysis of Arabidopsis transcription factor function. Plant J. 2011;68(3):560-9.

38. Nakashima K, Tran LSP, Van Nguyen D, Fujita M, Maruyama K, Todaka D, Ito Y, Hayashi N, Shinozaki K, Yamaguchi-Shinozaki K. Functional analysis of a NAC-type transcription factor OsNAC6 involved in abiotic and biotic stressresponsive gene expression in rice. Plant J. 2007;51(4):617-30.

39. AbuQamar S, Luo H, Laluk K, Mickelbart MV, Mengiste T. Crosstalk between biotic and abiotic stress responses in tomato is mediated by the AIM1 transcription factor. Plant J. 2009:58(2):347-60.

40. Lorenzo O, Piqueras R, Sánchez-Serrano JJ, Solano R. ETHYLENE RESPONSE FACTOR1 integrates signals from ethylene and jasmonate pathways in plant defense. The Plant Cell Online. 2003;15(1):165-78.

41. Anderson JP, Badruzsaufari E, Schenk PM, Manners JM, Desmond OJ, Ehlert C, Maclean DJ, Ebert PR, Kazan K. Antagonistic interaction between abscisic acid and jasmonate-ethylene signaling pathways modulates defense gene expression and disease resistance in Arabidopsis. Plant Cell. 2004;16(12): 3460-79.

42. Pré M, Atallah M, Champion A, De Vos M, Pieterse CM, Memelink J. The AP2/ERF domain transcription factor ORA59 integrates jasmonic acid and ethylene signals in plant defense. Plant Physiol. 2008:147(3):1347-57.

43. Kazan K, Manners JM. Linking development to defense: auxin in plantpathogen interactions. Trends Plant Sci. 2009;14(7):373-82.

44. Quidde T, Osbourn A, Tudzynski P. Detoxification of a-tomatine byBotrytis cinerea. Physiol Mol Plant Pathol. 1998;52(3):151-65.

45. Wan C-Y, Wilkins TA. A modified hot borate method significantly enhances the yield of high-quality RNA from cotton (Gossypium hirsutum L.). Anal Biochem. 1994;223(1):7-12.

46. Zhong S, Joung J-G, Zheng Y, Y-r C, Liu B, Shao Y, Xiang JZ, Fei Z, Giovannoni JJ. High-throughput illumina strand-specific RNA sequencing library preparation. Cold Spring Harbor Protocols. 2011;(8) pdb. prot5652

47. Grabherr MG, Haas BJ, Yassour M, Levin JZ, Thompson DA, Amit I, Adiconis X, Fan L, Raychowdhury R, Zeng Q. Full-length transcriptome assembly from RNA-Seq data without a reference genome. Nat Biotechnol. 2011;29(7):644-52.

48. Zheng Y, Zhao L, Gao J, Fei Z. iAssembler: a package for de novo assembly of Roche-454/sanger transcriptome sequences. BMC bioinformatics. 2011; 12(1):453.

49. Ma C, Wang H, Macnish AJ, Estrada-Melo AC, Lin J, Chang Y, Reid MS, Jiang C-Z. Transcriptomic analysis reveals numerous diverse protein kinases and transcription factors involved in desiccation tolerance in the resurrection plant Myrothamnus flabellifolia. Horticulture Research. 2015;2:15034.

50. Pei H, Ma N, Tian J, Luo J, Chen J, Li J, Zheng Y, Chen X, Fei Z, Gao J. An NAC transcription factor controls ethylene-regulated cell expansion in flower petals. Plant Physiol. 2013;163(2):775-91.

51. Wu L, Ma N, Jia Y, Zhang Y, Feng M, Jiang C-Z, Ma C, Gao J. An ethyleneinduced regulatory module delays flower senescence by regulating cytokinin content. Plant Physiol. 2016:01064.02016.

52. Fu Y, Esselink GD, Visser RG, van Tuyl JM, Arens P. Transcriptome analysis of Gerbera hybrida including in silico confirmation of defense genes found. Front Plant Sci. 2016;7:247.

Ready to submit your research? Choose BMC and benefit from:

- fast, convenient online submission

- thorough peer review by experienced researchers in your field

- rapid publication on acceptance

- support for research data, including large and complex data types

- gold Open Access which fosters wider collaboration and increased citations

- maximum visibility for your research: over $100 \mathrm{M}$ website views per year

At BMC, research is always in progress.

Learn more biomedcentral.com/submissions 